\title{
Mobile accessibility
}

\author{
Daniel Gonçalves • Markel Vigo • Luís Carriço
}

Published online: 22 September 2013

(C) Springer-Verlag Berlin Heidelberg 2013

Recent years have witnessed an increasing importance of mobile devices. They pervade people's everyday lives, not only just in the form of feature phones, but also as smartphones and tablets. Some of these have the computing power of small computers. We are, thus, at the cusp of a fundamental change in how we relate to information and others, accessible at all times and places with the help of mobile devices.

Alas, this change might be barred to a large number of people, suffering from a wide range of disabilities. Mobile devices are increasingly visual, making them hard to use by the blind or other vision-impaired people. Keyboards are steadily being replaced by touch screens, without tactile feedback, introducing additional barriers. Users with limited mobility of their limbs are hardly able to pick up the devices or use their fingers for precise pointing. Additionally, mobile devices are being used as mediators to reach distal interfaces (e.g. interactive TV, information kiosks) providing people with disabilities with the means to potentially overcome physical barriers and freely interact with the environment.

Moreover, the mobile aspect often creates situations where those interaction modes are cumbersome or socially unacceptable, even for non-impaired people. Riding a bicycle on a mountain trail while looking at a map at the same time is both difficult and dangerous. Timely

D. Gonçalves $(\bowtie)$

INESC-ID, University of Lisbon, Lisbon, Portugal

e-mail: daniel.goncalves@inesc-id.pt

M. Vigo

University of Manchester, Manchester, UK

L. Carriço

LaSIGE, University of Lisbon, Lisbon, Portugal responding to urgent and confidential solicitations on a public site may be distressing or embarrassing. These are considered situational disabilities that often introduce similar problems for the use of mobile apparatus. Overall, the list of problems is immense. Fortunately, there is a nascent area of research, mobile accessibility, where researchers are focusing on solving accessibility problems in mobile devices and settings.

This special issue collects the extended and revised versions of the best papers that were presented at the Mobile Accessibility Workshop 2011. The papers are representative of the aforementioned areas of interest, focusing on the following: gestures as a way to overcome accessibility problems experienced when using speech dialogue systems; sound, in the context of emergency calls made by the deaf; the problems related to requiring visual feedback for text entry in mobile devices in a situated impairment perspective; and the use of touch screen devices by motor-impaired users.

Multimodality as the means to facilitate interaction with mobile applications is explored in "Using Hand Gestures to Control Mobile Spoken Dialogue System" by Nikos Tsourakis. In particular, this paper explores the issue of interacting with a speech dialogue system through device gestures, where the gestures are employed as commands to a language learning software. The authors propose six gestures which are accurately identified by machine learning algorithms, and users find easy to use. User tests with three individuals with different abilities (cerebral palsy, blind, elderly) corroborate a high-gesture detection accuracy and indicate positive attitudes towards using the system.

An iconographic mobile interface is presented in "SOSPhone: a mobile application for emergency call" by Hugo Paredes, Benjamim Fonseca, Miriam Cabo, Tania 
Pereira and Filipe Fernandes. SOSPhone allows deaf users to establish non-auditory communications with an emergency centre. The functionalities of the system help users building and delivering emergency texts in a semiautomatic way. User tests show that SOSPhone is not only useful for deaf users, but also for able-bodied individuals.

Hugo Nicolau, Tiago Guerreiro, David Lucas and Joaquim Jorge studied how that lack of feedback impacts text entry, when the users are walking and unable to provide continuous visual attention to their devices. They performed two sets of user studies. The first assesses the impact of walking on text entry. They found that people mostly ignore audio feedback and find traditional assistive technologies slow, and try to compensate by reducing walking speed instead (while still taking longer and committing more errors). Based on these results, they tried to optimize visual feedback to be more amenable to the divided attention. They used pre-attentive attributes such as key colour and size, the first of which was shown to improve the results found in the first evaluation.

Most touch screen devices have pre-determined interfaces, adapted for the average user. However, their flexibility allows custom interfaces to be used, better adapting to particular needs. This is the idea explored by Hugo Nicolau, Tiago Guerreiro, Joaquim Jorge, Daniel Gonçalves, in relation to motor-impaired users. They present an evaluation performed with 15 tetraplegic and 18 ablebodied users that allowed to identify their main similarities and differences within a set of interaction techniques (tapping, crossing and directional Gesturing). They found that tapping and crossing work well for both types of users, while directional gesturing is not well suited for the former.
The results allowed them to find a set of guidelines for the design of inclusive touch interfaces, including preferred gestures, target sizes and screen position of targets.

The editors of this special issue would like to thank the authors for their relevant contributions for an emerging area that will ultimately improve universal access to information and services ubiquitously. We would also like to thank the Editorial Board of the International Journal Universal Access in the Information Society, and the reviewers for their thorough and constructive feedback that resulted in improved versions of these articles.

\section{List of reviewers}

Amaia Aizpurua, University of the Basque Country, Spain Borja Bonail, Tecnalia, Spain

Giorgio Brajnik, University of Udine, Italy

Andrew Brown, University of Manchester, UK

Stefan Carmien, Tecnalia, Spain

Alex Chen, University of Manchester, UK

Tiago Guerreiro, University of Lisbon, Portugal

Caroline Jay, University of Manchester, UK

Rui Lopes, Google, USA

Hugo Nicolau, Technical University of Lisbon, Portugal

Peter Thiessen, eBuddy, Netherlands

Markku Turunen, University of Tampere, Finland

Zigor Salvador, University of the Basque Country, Spain

Yeliz Yesilada, METU NCC, North Cyprus

Gottfried Zimmermann, Stuttgart Media University, Germany 